\title{
Análisis de Redes Sociales como estrategia para estudiar los Sistemas de Innovación. Revisión sistemática de la literatura*
}

\author{
Cesar Acevedo-Argüello \\ Sergio Zabala-Vargas ${ }^{2}$ \\ Julio Rojas-Mesa ${ }^{3}$ \\ Oscar Guayán-Perdomo ${ }^{4}$
}

Recibido: 14-06-2019

Aceptado: 13-03-2020

\section{Resumen}

Este documento está orientado a la construcción de un análisis bibliométrico y una revisión sistemática de la literatura sobre la aplicación del análisis de redes sociales como método de estudio de los sistemas de innovación. La revisión se llevó a cabo en una ventana de observación de 2001 a 2017. Se realizó la búsqueda en los índices bibliográficos Scopus y Web of Science Core Collection. Se obtuvo un conjunto de 1091 registros, con los cuales se realizó

\footnotetext{
* El artículo hace parte de procesos de investigación del Doctorado en Educación de la Universidad Santo Tomás. La tipología del artículo es de revisión. El documento es derivado parcialmente presentado como monografía del trabajo de maestría presentado en (Acevedo-Argüello et al., 2018)
}

1. Universidad Santo Tomás Seccional Bucaramanga. Centro de Recursos para el Aprendizaje y la Investigación.

Correo electrónico: cesar.acevedo@ustabuca.edu.co ORCID: https://orcid.org/0000-0002-0979-4113

2. Universidad Santo Tomás Seccional Bucaramanga. Facultad de Ingeniería de Telecomunicaciones.

Correo electrónico: sergio.zabala@ustabuca.edu.co ORCID: https://orcid.org/0000-0001-5803-1123

3. Universidad Santo Tomás Seccional Bogotá. Doctorado en Educación. Correo electrónico: juliorojas@usantotomas.edu.co ORCID: http://orcid.org/0000-0002-2237-3727

4. Universidad Santo Tomás Seccional Bogotá. Doctorado en Educación. Correo electrónico: oscarguayan@gmail.com

ORCID: https://orcid.org/0000-0001-8952-9899 
un análisis bibliométrico de la actividad, la relación y el impacto. Por otro lado, se llevó a cabo la selección de 29 artículos recurriendo a criterios de inclusión específicos, permitiendo construir categorías asociadas a patentes, redes de coautoría, información de proyectos conjuntos, combinaciones de fuentes de datos, entre otros.

Palabras clave: Análisis de Redes Sociales; Sistemas de Innovación; Indicadores bibliométricos; Análisis de Contenido; Revisión sistemática de la literatura.

\title{
Social Networks Analysis as a tool to study Innovation Systems. A Systematic review
}

\begin{abstract}
This paper presents a bibliometric analysis and a systematic review of the literature on the application of social network analysis as a method to study innovation systems. The review was made from 2001 to 2017. The search was made in the bibliographic indexes: Scopus and Web of Science Core Collection. A set of 1091 records was obtained, in which a bibliometric analysis of the activity, the relationship and the impact was carried out. On the other hand, the selection of 29 articles was made using specific inclusion criteria, which allowed the construction of categories associated with patents, co-authorship networks, information on joint projects, combinations of data sources, among others.
\end{abstract}

Keywords: Social Networks Analysis; Innovation Systems;

Bibliometric Indicators; Content Analysis; Systematic review. 


\section{Introducción}

El concepto de innovación y su importancia ha sido ampliamente discutido en la literatura. Formalmente, la innovación, como se propone en el Manual de Oslo, es "la concepción o implementación de cambios significativos en productos, procesos, mercados, organización empresarial o relaciones externas" (OECD/Eurostat, 2005); con el propósito de mejorar los resultados de las organizaciones y los países.

Coherente con lo anterior, la Organización para la Cooperación y el Desarrollo Económico-OECD (2014) cita que la innovación sustenta el crecimiento y el dinamismo de todas las economías, convirtiéndose en uno de los principales motores para el desarrollo de los países, de tal manera que la innovación contribuye con cerca del $50 \%$ del total de su crecimiento económico, medido a través del Producto Interno Bruto-PIB. Indica también la OCDE que uno de los aspectos claves para el desarrollo y la innovación se encuentra en la interrelación e involucramiento de un amplio espectro de actores, entre los que se destacan empresas, fundaciones, organizaciones sin ánimo de lucro, universidades, institutos científicos, agencias del sector público, ciudadanos, consumidores; siempre trabajando en una colaboración muy cercana. Ampliando este concepto de innovación; países, regiones, ciudades y organizaciones han buscado fortalecer ecosistemas que se traduzcan en Sistemas de Innovación eficientes. Los Sistemas de Innovación abarcan organizaciones que se relacionan en el marco de un conjunto de instituciones que las regulan y orientan (Edquist, 2006).

Dicha faceta sistémica está constituida por las interacciones de las organizaciones (de manera puntual, aunque no restrictiva: universidades, centros de investigación, empresas de diferentes actividades económicas, e instituciones públicas), hecho que hace relevante la búsqueda de enfoques y herramientas metodológicas que permitan interpretar la eficiencia de dichas relaciones para el conjunto del sistema. Los estudios acerca de los sistemas de innovación han adoptado la aplicación de una metodología de inves- 
tigación particular denominada Análisis de Redes Sociales, principalmente orientada a facilitar el flujo del conocimiento como insumo de la innovación a través de las redes de colaboración con estructuras que facilitan tal proceso (Van Der Valk y Gijsbers, 2010).

En la presente revisión sistemática de la literatura se analizaron los referentes seleccionados mediante una metodología de búsqueda de información aplicada en bases de datos de corriente principal (Scopus y Web of Science Core Collection) relacionados con Análisis de Redes Sociales y Sistemas de Innovación publicados entre 2001 y 2017. Tales registros fueron analizados para obtener indicadores bibliométricos con el fin de caracterizar las dinámicas de investigación y difusión de resultados de investigación al respecto. Mediante la aplicación de criterios de exclusión e inclusión, se revisaron 29 artículos que, por medio del análisis de su contenido, fueron analizados para clasificarlos según una tipología surgida a partir de las metodologías de los estudios mencionados.

El objetivo específico de este documento es poder compartir con la comunidad académica la construcción de una tipología a partir de las aplicaciones documentadas del Análisis de Redes Sociales como método de estudio de los Sistemas de Innovación mediante la revisión sistemática y el análisis bibliométrico del corpus de textos publicados en fuentes indexadas en bases de datos bibliográficas de corriente principal, con el fin de evaluar sus alcances y resultados. Los conceptos básicos en los que se fundamenta el presente estudio se encuentran documentados en dos fuentes de literatura científica, a saber: los métodos del Análisis de Redes Sociales y la investigación acerca de Sistemas de Innovación. Desde el punto de vista metodológico en el presente estudio se aplican los lineamientos relacionados con revisiones sistemáticas de la literatura en ciencias sociales, análisis de indicadores bibliométricos, y análisis de contenido (Gast, Schildkamp, y Veen, 2017; Petticrew y Roberts, 2006). A continuación, se definen tales conceptos de base de acuerdo con la literatura clave en los asuntos mencionados. 


\section{Marco de referencia}

Para empezar, se hace necesario definir el concepto de Sistema de Innovación. De acuerdo con Charles Edquist (2006), un Sistema de Innovación está conformado por todos los factores (económicos, sociales, políticos, organizacionales, entre otros) que influyen en el desarrollo, difusión y uso de innovaciones. Tales factores pueden funcionar y coexistir en niveles nacionales, regionales y sectoriales (Edquist, 2006; Schiller, 2015). En coherencia con lo anterior, Edquist concibe a organizaciones e instituciones como los componentes principales de Sistemas de Innovación. Las organizaciones se definen como estructuras formales creadas de forma consciente, tales como las empresas, las universidades y las agencias públicas. Asimismo, las instituciones pertenecen al terreno de las regulaciones que rigen las relaciones de las organizaciones (Edquist, 2006).

A su vez, el carácter sistémico de la innovación según este enfoque, implica que de las interacciones de las organizaciones del sistema depende su desempeño innovador (Doloreux y Porto Gomez, 2017), de manera que la innovación no surge de forma aislada desde las organizaciones que lo conforman (Stuck y otros, 2016). Cabe anotar que estas posturas teóricas acerca de los sistemas de innovación se sustentan en una economía de la producción, uso y difusión del conocimiento (Lundvall, 2007).

En relación con las conceptualizaciones anteriores, uno de los modelos de estudio identificados en la literatura de Sistemas de Innovación se denomina Triple Hélice, propuesto por Henry Etzkowitz y Loet Leydesdorff (Dolfsma y Leydesdorff, 2011). Según este modelo, existen tres actores principales, a saber, las Universidades, la Empresa y las Instituciones públicas, cuyas interacciones sirven para explicar los cambios en economías basadas en el conocimiento. Cabe aclarar, que el vínculo de la noción de Sistemas de Innovación, y el modelo de la Triple Hélice, podría prescindir de la condición ex ante de la delimitación geográfica en favor del análisis de las sinergias de las hélices postuladas (Dolfsma y Leydesdorff, 2011; Smith y Leydesdorff, 2014). 
Por su parte, tal como lo proponen Smith y Leydesdorf (2014), el Análisis de Redes Sociales podría enriquecer el modelo de la Triple Hélice como instrumento para la formulación de políticas de creación de redes. De acuerdo con Pinheiro y otros (2015) un supuesto básico para el análisis de redes sociales es que hay interdependencia en las actividades de los miembros de una red y con el entorno que enmarca a la mencionada red, de manera que su estructura aporta oportunidades y limitaciones a sus miembros. En tal sentido, la interacción de los miembros de la red influye tanto como los atributos individuales de los actores (Pinheiro y otros, 2015); y es capaz de potenciar círculos virtuosos de innovación que fomenten el desarrollo social y económico de los países (Davyt y Mujica, 2011).

De acuerdo con de Souza y otros (2015), el Análisis de Redes Sociales (Social Network Analysis) surge de la teoría de grafos. Un grafo es el equivalente de una red, en la cual se reconocen nodos (personas, organizaciones, conglomerados que trabajan para el logro de un objetivo común); aristas, definidas como interacciones o vínculos entre nodos; y flujos, es decir, la direccionalidad de la relación. Por su parte, para el análisis de la red se reconocen indicadores que permiten entender la intensidad de las relaciones, tales como el grado de centralidad (degree centrality), intermediación (betweenness), la centralidad eigenvector (eigenvector centrality), la densidad de la red (network density), entre otros (Swar y Khan, 2013).

De este modo, la metodología de la revisión sistemática se basa en el análisis de las publicaciones relevantes para un asunto de investigación de acuerdo con una pregunta guía y con base en una metodología de búsqueda, selección y síntesis de documentos (Petticrew y Roberts, 2006). Con el fin de clasificar los artículos revisados, se recurrió al análisis de contenido por medio de la clasificación en categorías permitiendo elaborar una tipología de las aplicaciones del análisis de redes sociales a sistemas de innovación (Fernández Chaves, 2002)

Finalmente, los indicadores bibliométricos proveen información cuantitativa acerca de la productividad, la colaboración y el impac- 
to de la actividad de investigadores reflejada en sus publicaciones. Este tipo de datos, aunque representan tan sólo una dimensión de la actividad científica, resultan útiles para la toma de decisiones en asuntos como la evaluación y la administración de actividades de ciencia, tecnología e innovación (Galeano y otros, 2018; Waltman y Noyons, 2018)

\section{Materiales y métodos}

La metodología de revisión sistemática aplicada en el presente estudio realiza una adaptación a lo expuesto por Petticrew y Roberts (2006) adicionando el análisis bibliométrico de la información; como lo sugiere (Gast y otros, 2017). Los pasos propuestos son:

\section{Preguntas de investigación}

En esta fase se realizó la definición de las preguntas específicas de investigación para la revisión sistemática. Las preguntas fueron: ¿Qué relación se encuentra en la literatura entre el concepto de análisis de redes sociales y los sistemas de innovación?; ¿Cuáles son las metodologías e instrumentos utilizados en las investigaciones encontradas en la revisión?; ¿Cuáles son las principales categorías o dimensiones del relacionamiento de los conceptos principales de la revisión (análisis de redes sociales y sistemas de innovación)?

\section{Definición de bases de datos}

\section{e índices bibliográficos para la revisión}

Debido a la pertinencia temática y a la robustez de los índices Scopus y Web of Science (WoS); fueron estos los seleccionados para la revisión. A su vez se incorporó para el tratamiento de la información y la minería de datos el software Vantage Point 10. 


\section{Términos de búsqueda de registros - Ecuación de búsqueda}

Los principales términos seleccionados para la revisión sistemática de la literatura son: 1) Sistemas de Innovación y 2) Análisis de redes sociales. Cada uno de estos términos es soportado a través de sinónimos o términos clave similares, que permitan aumentar el rango de la búsqueda; así como el registro de estos en idioma inglés. La ecuación de búsqueda finalmente utilizada (ejemplo caso WoS) fue:

$T S=((" I n n o v a t i o n$ System $*$ " OR "National Innovation System*" OR "Regional Innovation System*" $O R$ innovator* $O R$ "research and development" $O R$ "R\&D" $O R$ "university-industry-government" $O R$ "triple helix" $O R$ "university-industry" OR "public private") AND ("social network* analysis" $O R$ "collaboration network*" OR proximity $O R$ centrality $O R$ "Ego network*" OR "Ego-network*" OR "Co-author*" OR "Co author")).

El número de registros obtenidos fue: 715 Scopus y 779 WoS, para un total de 1494 registros bibliográficos. El detalle de las ecuaciones de búsqueda se encuentra visible en (Acevedo-Argüello y otros, 2018)

\section{Definición y aplicación de criterios de inclusión-preprocesamiento de datos}

Los criterios de inclusión en la primera iteración realizada fueron:

a) Textos completos o resúmenes de artículos de investigación, comunicaciones en conferencias, capítulos de libros y libros en los que se apliquen los métodos del análisis de redes sociales a sistemas de innovación.

b) Ventana de observación de 2001 a 2017.

c) Fuentes indexadas en Scopus y en la Colección Principal de Web of Science. Cabe resaltar que el artículo se incluye si aparece en alguna de las dos bases de datos 
mencionadas, no necesariamente debe aparecer en las dos.

Estos fueron aplicados sobre el corpus de conocimiento obtenido del paso anterior. Además, se llevó a cabo un preprocesamiento de datos bibliográficos mediante el software de minería de textos Vantagepoint 10. Con esto se logró la eliminación de duplicados, errores en el registro de autores, filiaciones y países. Luego de este procesamiento se obtuvieron, en total, 1091 registros bibliográficos.

\section{Análisis bibliométrico}

El corpus de conocimiento consolidado a partir de las fuentes consultadas y preprocesado para la minería de textos en el software Vantagepoint 10, fue analizado cuantitativamente para obtener los indicadores detallados a continuación:

a) Frecuencia de publicación por años.

b) Frecuencia de publicación por países, instituciones y autores.

c) Frecuencia de publicación por revistas científicas.

d) Frecuencia de publicación por asuntos de investigación de acuerdo con las palabras clave.

e) Indicadores de relación- Publicaciones de resultados de investigación en colaboración y redes de coautoría.

\section{Aplicación de criterios de inclusión específicos- selección de documentos de la revisión sistemática}

Para ello, se categorizaron los documentos de acuerdo con temáticas generales ajustadas al tema de la revisión y se obtuvo una matriz con los artículos pertinentes que de nuevo fueron evaluados a la luz de los criterios de inclusión y exclusión. Particularmente para la revisión sistemática se consideraron aquellos artículos asociados 
a descriptores de Análisis de Redes Sociales, relacionados con los descriptores propuestos de sistemas de Innovación.

Esta aplicación de etapas sucesivas para la revisión sistemática como lo sugiere (Petticrew y Roberts, 2006); permitió depurar el corpus de conocimiento a 29 documentos para la revisión final. Estos 29 documentos fueron el resultado del control de calidad, la calidad de los artículos se verificó utilizando 11 criterios de calidad extraídos de Petticrew y Roberts (2006) adaptado por Gast et al. (2017), los cuales se presentan en la Tabla I. Cada criterio se evaluó en tres escalas: 1) 0 , no está definido, 2) 0.5 , se presenta, pero no con total claridad y 3) 1, se presenta claramente. Para ser incluidos en la revisión sistemática, los artículos debían tener una puntuación de al menos 5.5 para los 11 criterios, al menos la mitad de la cantidad máxima de puntos posibles.

Tabla I. Criterios de Calidad (Adaptada de Gast et al., 2017).

\begin{tabular}{|c|c|}
\hline Categoría & Criterio de calidad \\
\hline \multirow{2}{*}{ General } & 1. ¿Está claro el objetivo de la investigación? \\
\hline & $\begin{array}{l}\text { 2. ¿La investigación realizada con el método elegido es capaz de encontrar una } \\
\text { respuesta clara a la pregunta de investigación? }\end{array}$ \\
\hline \multirow{2}{*}{$\begin{array}{l}\text { Muestra de } \\
\text { selección }\end{array}$} & 3. ¿Se reunieron suficientes datos para asegurar la validez de las conclusiones? \\
\hline & 4. ¿Está claro el contexto de la investigación (país, participantes)? \\
\hline \multirow{3}{*}{ Método } & 5. ¿Indican los investigadores los métodos de investigación utilizados? \\
\hline & 6. ¿Los autores dan un argumento para los métodos elegidos? \\
\hline & $\begin{array}{l}\text { 7. ¿Los investigadores toman en cuenta otras variables que podrian ser de } \\
\text { influencia? }\end{array}$ \\
\hline \multirow{3}{*}{ Análisis de datos } & 8. ¿Se analizan los datos de manera adecuada y precisa? \\
\hline & 9. ¿Están los resultados claramente presentados? \\
\hline & $\begin{array}{l}\text { 10. ¿Los investigadores informan sobre la confiabilidad y validez de la } \\
\text { investigación? }\end{array}$ \\
\hline Conclusión & $\begin{array}{l}\text { 11. ¿Se responde a la pregunta de investigación utilizando evidencia empírica de } \\
\text { la investigación que se realizó? }\end{array}$ \\
\hline
\end{tabular}

Fuente: elaboración propia. 
Los artículos incluidos finalmente se revisaron de forma crítica desde el punto de vista de las variantes metodológicas asociadas a la aplicación del Análisis de Redes Sociales a Sistemas de Innovación; realizando una clasificación y permitiendo generar respuestas a las preguntas de investigación, categorías o dimensiones del análisis y conclusiones del proceso.

\section{Resultados y discusión}

Para empezar, se analizó de manera bibliométrica un corpus unificado de 1091 registros a partir de las fuentes de información bibliográfica. Los indicadores a continuación permiten interpretar las tendencias en la publicación de resultados de investigación asociados al tema de revisión, de acuerdo con el periodo estudiado, los países de afiliación y las instituciones, los autores, las revistas científicas, y las temáticas de investigación rastreadas mediante los descriptores clave incluidos en los artículos.

\section{Indicadores bibliométricos de}

\section{actividad-Frecuencia de publicación por años}

De acuerdo con los datos de la frecuencia de publicación por años se puede evidenciar que entre 2001 y 2010 se publicó el $25.76 \%$ de los documentos en el corpus. Entre 2011 y 2017 se duplicó la cantidad de publicaciones, lo cual indica un interés creciente en el tema de investigación. Esta tendencia guarda relación con los resultados de Van der Valk y Gijsbers (2010). En la Figura 1 se presentan los resultados cada dos años. 
Figura 1. Frecuencia de publicación por año.

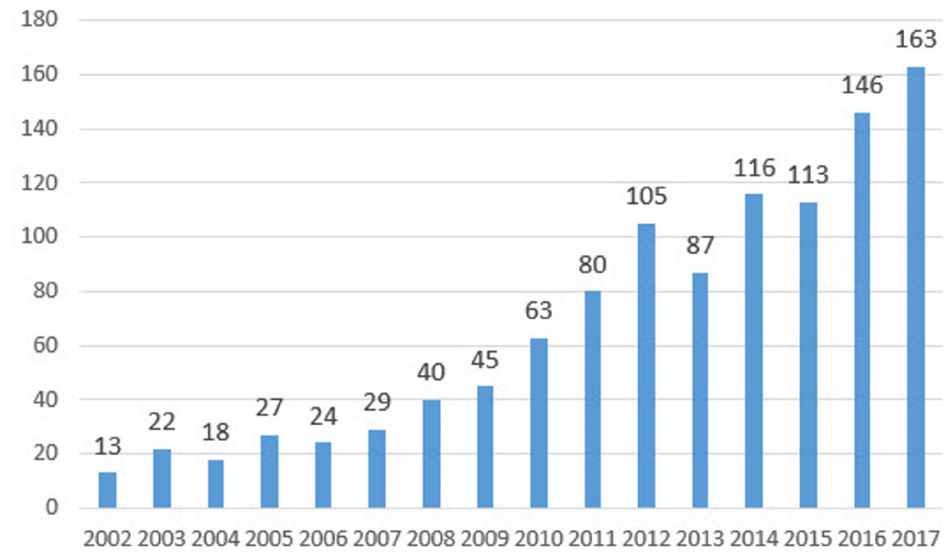

Fuente: Elsevier (2018) y Clarivate Analytics (2018). Fecha de consulta: 5 de junio de 2018.

\section{Indicadores bibliométricos de}

actividad-Frecuencia de publicación

por países, instituciones y autores

En esta sección se presenta, en resumen, la frecuencia de publicación de acuerdo con los países de afiliación con mayor cantidad de publicaciones. En conjunto, los diez países relacionados en la Figura 2 registran el $85.79 \%$ de las publicaciones del análisis bibliométrico. Se resalta a Estados Unidos, Gran Bretaña e Italia como los países con mayor cantidad de registros.

Figura 2. Relación de número de registros por país (10 primeros).

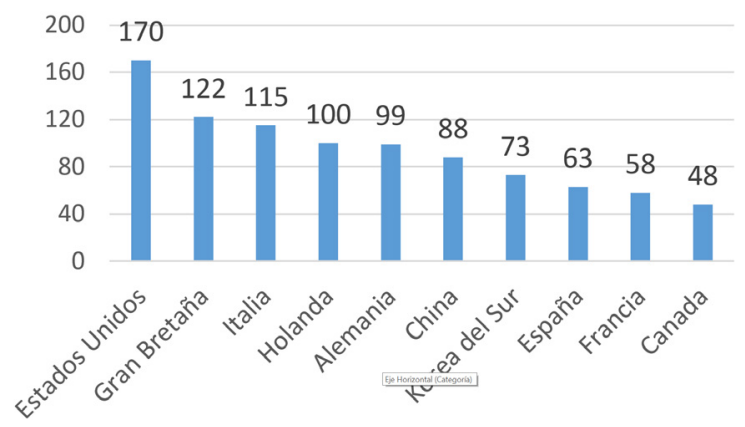

Fuente: Elsevier (2018) y Clarivate Analytics (2018). Fecha de consulta: 5 de junio de 2018. 
Ahora bien, en la Tabla II se presentan las instituciones (afiliaciones) con mayor número de registros. En este caso es importante resaltar los aportes que sobre la temática ha realizado Utrecth University (Holanda) con 24 registros, la Univ. Jena (Alemania) con 16 registros y University of Ámsterdam (Holanda) con 15.

Tabla II. Número de registros por institución/afiliación (mayores a 9).

\begin{tabular}{|l|c|}
\hline Institución & \# de registros \\
\hline Utrecht University, Netherlands & 16 \\
\hline Univ. Jena, Jena, Germany & 15 \\
\hline University of Amsterdam, Netherlands & 14 \\
\hline Universidad Politécnica de Valencia, Spain & 12 \\
\hline Leibniz-Universität Hannover, Germany & 11 \\
\hline Erasmus University Rotterdam, Netherlands & 11 \\
\hline McGill Univ, Montreal PQ, Canada & 11 \\
\hline Seoul National University, South Korea & 11 \\
\hline Tilburg Univ, Tilburg, Netherlands & 10 \\
\hline Bocconi Univ, Milan, Italy & 10 \\
\hline London Sch Econ, London, England & 10 \\
\hline University of Chinese Academy of Sciences, China & 9 \\
\hline Cardiff Univ, S Glam, Wales & 9 \\
\hline Free Univ Berlin, Berlin, Germany & 9 \\
\hline Univ Ottawa, Ottawa ON, Canada & 14 \\
\hline Univ Sussex, E Sussex, England & 11 \\
\hline
\end{tabular}

Fuente: Elsevier (2018) y Clarivate Analytics (2018). Fecha de consulta: 5 de junio de 2018.

Finalmente, en la Tabla III se presenta una relación de autores, los cuales cuentan con la mayor productividad (medida por el número de registros) en la ventana de observación. En esta tabla se relacionan los autores con más de 6 publicaciones. 
Tabla III. Relación de autores con mayor productividad en la temática (mayores a 6).

\begin{tabular}{|l|c|}
\hline Autor & \# de registros \\
\hline Leydesdorff, Loet & 13 \\
\hline Park, Han Woo & 9 \\
\hline Shearmur, Richard & 8 \\
\hline Frenken, Koen & 7 \\
\hline van Oort, Frank & 7 \\
\hline Broekel, Tom & 7 \\
\hline Guan, Jiancheng & 7 \\
\hline Wang, Yuandi & 6 \\
\hline Huggins, Robert & 6 \\
\hline Rodriguez-Pose, Andres & 6 \\
\hline Doloreux, David & 11 \\
\hline
\end{tabular}

Fuente: Elsevier (2018) y Clarivate Analytics (2018). Fecha de consulta: 5 de junio de 2018.

\section{Frecuencia de publicación por revistas científicas}

Las fuentes secundarias de mayor peso en el corpus bibliográfico son revistas científicas que difunden los resultados de investigaciones asociados a la evaluación de actividades de I+D+i, economía y administración, y la gestión tecnológica. En la Tabla IV se presentan las revistas científicas con mayor cantidad de registros (acotada a aquellas con más de 14 registros)

Tabla IV. Relación de revistas científicas con mayor número de registros (mayores a 10).

\begin{tabular}{|l|c|c|}
\hline Publicaciones & \# de registros & ISSN \\
\hline Scientometrics & 68 & $0138-9130$ \\
\hline Research Policy & 52 & $0048-7333$ \\
\hline European Planning Studies & 44 & $1469-5944$ \\
\hline Regional Studies & 43 & $0034-3404$ \\
\hline
\end{tabular}




\begin{tabular}{|c|c|c|}
\hline Publicaciones & \# de registros & ISSN \\
\hline Journal of Technology Transfer & 22 & 0892-9912 \\
\hline Papers in Regional Science & 21 & $1056-8190$ \\
\hline Technological Forecasting and Social Change & 20 & $0040-1625$ \\
\hline Technovation & 16 & 0166-4972 \\
\hline Annals of Regional Science & 14 & $1432-0592$ \\
\hline Industry and Innovation & 14 & $1469-8390$ \\
\hline R \& D Management & 14 & $1467-9310$ \\
\hline International Journal of Technology & 13 & $1433-3015$ \\
\hline Journal of Economic Geography & 13 & $1468-2702$ \\
\hline Economics of Innovation and New Technology & 11 & $1476-8364$ \\
\hline Science and Public Policy & 10 & $1471-5430$ \\
\hline Strategic Management Journal & 10 & $1097-0266$ \\
\hline
\end{tabular}

Fuente: Elsevier (2018) y Clarivate Analytics (2018). Fecha de consulta: 2 de marzo de 2019. Información adicional (ISSN) tomada de https://www.scimagojr.com/

\section{Frecuencia de publicación por temáticas de investigación, acorde con palabras clave}

Con el fin de analizar el contenido de los registros, se combinaron los campos que contienen descriptores temáticos de ambas bases de datos y se desagregaron los títulos de los registros en frases del lenguaje natural. Esto permitió general un listado que cubre al $100 \%$ de los registros del corpus destinado al análisis bibliométrico. Dicho listado fue agrupado según 23 categorías con 2175 palabras claves. Una síntesis de estos elementos se presenta en la Tabla V.

Tabla V. Categorías de clasificación de términos (palabras claves).

\begin{tabular}{|l|c|}
\hline Categoria & \multicolumn{1}{c|}{ Ejemplo términos claves } \\
\hline Conocimiento & $\begin{array}{l}\text { Knowledge, Knowledge Transfer, Knowledge Management, Know-How, Knowledge } \\
\text { System, Knowledge Exchange, External Knowledge Sources, Diversified Knowledge. }\end{array}$ \\
\hline Universidad & $\begin{array}{l}\text { University, University Research, University Sector, Government-University-Industry } \\
\text { University Capability, University Entrepreneurial Orientation, University Incubators. }\end{array}$ \\
\hline
\end{tabular}




\begin{tabular}{|c|c|}
\hline Categoría & Ejemplo términos claves \\
\hline $\begin{array}{l}\text { Universidad- } \\
\text { Empresa }\end{array}$ & $\begin{array}{l}\text { Industry-University Collaboration, University-Industry Linkages, University-Industry } \\
\text { Research Collaboration, Academia-Industry, University-Business Cooperation. }\end{array}$ \\
\hline Empresas & $\begin{array}{l}\text { Firm, Industry, Firm Size, Firm Capabilities, Knowledge-Intensive Industry, Firm R\&D } \\
\text { Capabilities, Intrafirm Networks, Industry Collaboration. }\end{array}$ \\
\hline $\begin{array}{l}\text { Sistema } \\
\text { Nacional de } \\
\text { Innovación }\end{array}$ & $\begin{array}{l}\text { National Innovation System, Collaborative Invention Across National Innovation } \\
\text { Systems, National Innovation Cooperation, National Innovation Policies. }\end{array}$ \\
\hline $\begin{array}{l}\text { Sistema } \\
\text { Regional de } \\
\text { Innovación }\end{array}$ & $\begin{array}{c}\text { Regional Innovation System, Regional Innovation Policy, Regional Innovation } \\
\text { Capabilities, Regional Innovation Networks, Regional Innovation System Research. }\end{array}$ \\
\hline $\begin{array}{l}\text { Sistema } \\
\text { Sectorial de } \\
\text { Innovación }\end{array}$ & $\begin{array}{l}\text { Sectoral Patterns, Sectoral Differences, Sectoral Innovation System, Sectoral } \\
\text { Systems, Sectoral Innovativeness, Regional Sectoral Specialization. }\end{array}$ \\
\hline $\begin{array}{l}\text { Sistema Local } \\
\text { de Innovación. }\end{array}$ & $\begin{array}{c}\text { Local Innovation System, Local Innovation Activity, Tourism Local Innovation System, } \\
\text { Understanding Local Innovation Systems. }\end{array}$ \\
\hline Spin-off & $\begin{array}{c}\text { Spin-Off, Public Research Spin-Offs, Spin-Off Entrepreneurship, Spin-Off Smes, } \\
\text { University Spin-Offs. }\end{array}$ \\
\hline Start-ups & $\begin{array}{c}\text { Start-Up, High-Tech Start-Ups, Innovative Start-Up, Regional High-Tech Firm Start- } \\
\text { Up }\end{array}$ \\
\hline Colaboración & $\begin{array}{l}\text { Collaboration, Collaboration Network, Research Collaboration, Knowledge } \\
\text { Collaboration, Patent Collaboration, Team Collaboration, Collaboration Model. }\end{array}$ \\
\hline $\begin{array}{l}\text { Propiedad } \\
\text { Intelectual }\end{array}$ & $\begin{array}{l}\text { Patent, Patent Network, Co-Patent, Patent Collaboration, Patent Indicators, Patent } \\
\text { Citation Network Analysis, Patent Contests, Patent Information. }\end{array}$ \\
\hline Coautoría & $\begin{array}{l}\text { Coauthorship, Asymmetric Authorship, Scientific Co-Authorship, Joint Authorship, } \\
\text { University-Government-Enterprise Co-Authorship Networks, Multiple Authorship. }\end{array}$ \\
\hline Centralidad & $\begin{array}{l}\text { Network Centrality, Betweenness Centrality, Government Centrality, Global Network } \\
\text { Centrality, Organization Centrality, Firm's Centrality, Conflict Centrality. }\end{array}$ \\
\hline Tecnología & $\begin{array}{l}\text { Technology, Technology Transfer, High Technology Industry, Technology Cluster, New } \\
\text { Technology Based Firm, Technology Entrepreneurship, Technology Research. }\end{array}$ \\
\hline $\begin{array}{l}\text { Innovación } \\
\text { abierta }\end{array}$ & $\begin{array}{l}\text { Open Innovation, Open Innovation Communities, Coupled Open Innovation } \\
\text { Practices, Inbound Open Innovation, Open Innovation Processes. }\end{array}$ \\
\hline Innovación & $\begin{array}{l}\text { Innovation, Innovation System, Product Innovation, Innovation Performance, } \\
\text { Environmental Innovation, Innovation Output, Innovation Strategy. }\end{array}$ \\
\hline Cluster & $\begin{array}{l}\text { Cluster, Cluster Policy, Cluster Connectivity, Cluster Structures, Clustering } \\
\text { Algorithms, Knowledge-Intensive Technology Clusters. }\end{array}$ \\
\hline Proximidad & $\begin{array}{c}\text { Proximity, Social Proximity, Cognitive Proximity, Organizational Proximity, Knowledge } \\
\text { Proximity, Urban Geography. }\end{array}$ \\
\hline $1+D$ & $\begin{array}{l}\text { R\&D, Research, Research Collaboration, Academic Research, R\&D Collaboration, } \\
\text { R\&D Collaboration Network, Public Research Organizations, R\&D Activities. }\end{array}$ \\
\hline Spillover & $\begin{array}{l}\text { Spillover, Knowledge Spillover, Spillover Effect, Direct R\&D Spillovers, Distant } \\
\text { Knowledge Spillovers, Dynamic Knowledge Spillovers, Spillover Networks. }\end{array}$ \\
\hline $\begin{array}{l}\text { Análisis } \\
\text { bibliométrico }\end{array}$ & $\begin{array}{c}\text { Bibliometric, Bibliometric Analysis, Scientometrics, Scientometrics Study, Bibliometric } \\
\text { Assessments, Bibliometric Comparison, Bibliometric Tool, Patentometrics. }\end{array}$ \\
\hline
\end{tabular}

Fuente: Elsevier (2018) y Clarivate Analytics (2018). Fecha de consulta: 2 de marzo de 2019. Información adicional (ISSN) tomada de https://www.scimagojr.com/ 
Con estos elementos se generó una matriz de adyacencia, con lo cual fue posible analizar la coocurrencia de los descriptores seleccionados. Dicha matriz se representa, utilizando el software VOSviewer, cuyo algoritmo de análisis permite identificar clústeres de entidades de acuerdo con las coocurrencias y con su peso en términos de registros asociados; y se presenta en la Figura 3.

Figura 3. Análisis de coocurrencia de descriptores agrupados según categorías.

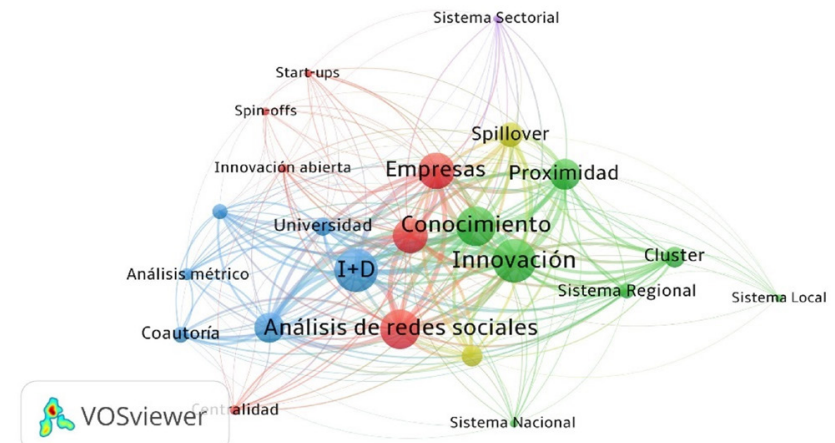

Fuente: Elsevier (2018) y Clarivate Analytics (2018). Fecha de consulta: 2 de marzo de 2019. Procesada con el software VOSviewer.

\section{Indicadores de relación- Publicación}

de resultados de investigación

en colaboración y redes de coautoría

Desde los registros revisados en la presente revisión se resalta que aquellos países con niveles de colaboración más altos son, también, los de mayor cantidad de publicaciones. En la Figura 4 se representa la red de colaboración, generada a partir de la matriz de adyacencia. En la parte central se observan los países con mayor productividad (Estados Unidos, Gran Bretaña, Corea del Sur, Holanda, Suecia e Italia), y hacia la periferia de la red los de menor colaboración. En la parte superior izquierda se relacionan los países aislados.

La Figura 4 también permite identificar la centralidad de los nodos en la red de coautoría, a través del grado de intermediación o 
betweenness centrality, definido como la frecuencia con la cual un nodo aparece en el camino más corto entre dos nodos diferentes (Borgatti y otros, 2013). En este sentido se resalta la relevancia, en el grado de intermediación, de las instituciones con registro en Estados Unidos y Gran Bretaña. En términos generales, se asume que el grado de intermediación alto significa potencial para el control de los flujos en la red (Borgatti y otros, 2013).

Figura 4. Representación de la red de coautoría por países de afiliación.

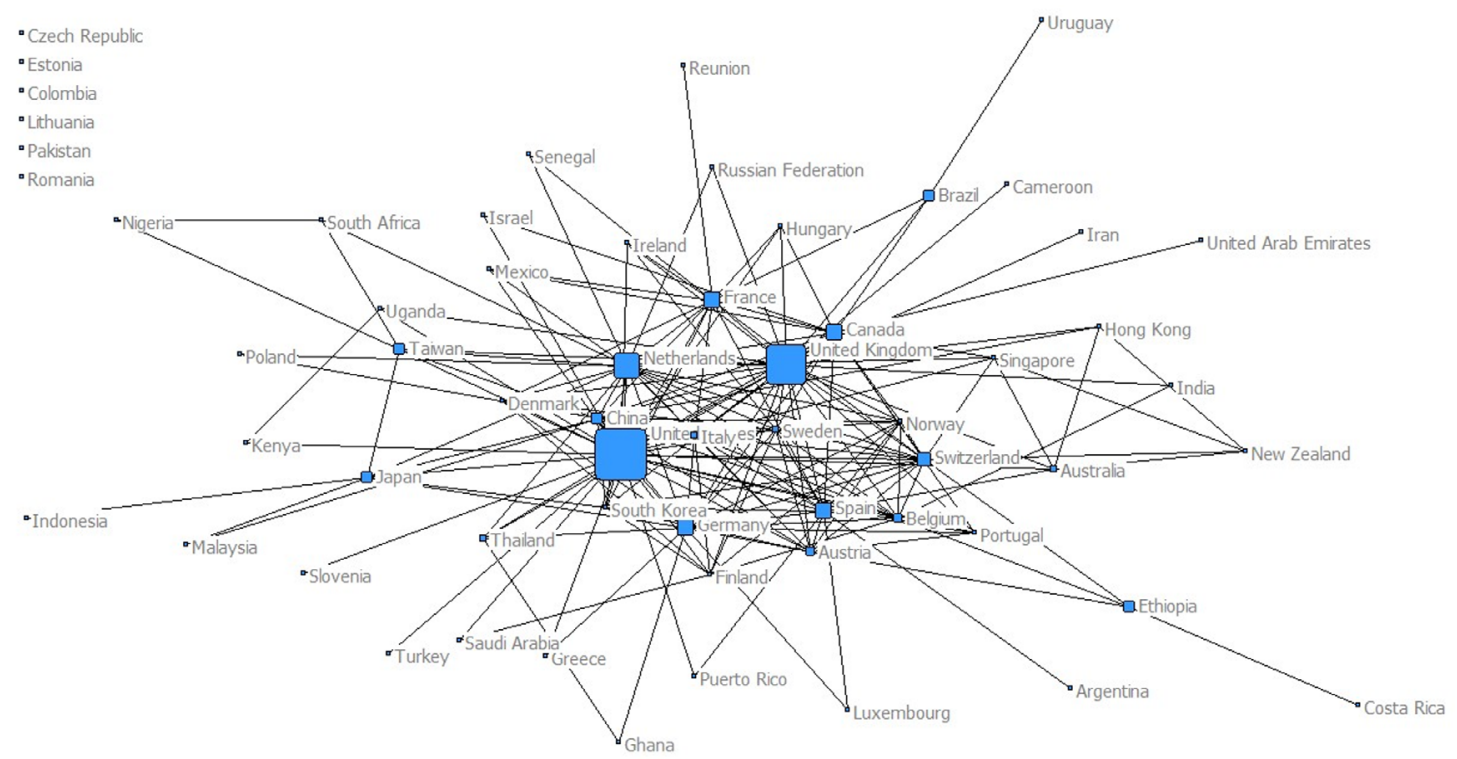

Fuente: Elsevier (2018) y Clarivate Analytics (2018). Fecha de consulta: 2 de marzo de 2019. Procesada con el software UCINET6.0.

\section{Análisis de la literatura- Categorización y discusión}

En la revisión de literatura realizada sobre los sistemas de innovación, se encuentra que la aplicación del método de análisis de redes sociales se lleva a cabo a partir de múltiples tipos de fuentes, objetivos de estudio y herramientas. En la Tabla VI se relaciona una síntesis global de las categorías obtenidas a partir de la revisión de los resúmenes y apartados metodológicos de los registros 


\section{identificados desde la matriz de coocurrencia; a su vez, siguiendo la metodología propuesta, se clasificaron los estudios de acuerdo con la fuente de información usada para analizar las redes sociales, el alcance de la categoría y una síntesis de resultados de estas.}

Tabla VI. Descripción de las categorías de análisis a partir de la revisión sistemática y artículos.

\begin{tabular}{|c|c|c|c|c|}
\hline Categoría & Contenido & Registros (Referencias) & Alcance & Resultados \\
\hline $\begin{array}{l}\text { Redes de } \\
\text { patentamiento }\end{array}$ & $\begin{array}{l}\text { Se } \\
\text { comprenden } \\
\text { las redes } \\
\text { según } \\
\text { los datos } \\
\text { asociados a } \\
\text { los titulares de } \\
\text { las patentes } \\
\text { de los actores } \\
\text { del sistema de } \\
\text { innovación. }\end{array}$ & $\begin{array}{l}\text { 1- (Balconi y otros, 2004). } \\
\text { 2- (Cantner y Graf, 2006). } \\
\text { 3- (Cantner y otros, 2010). } \\
\text { 4- (Dolfsma y Leydesdorff, 2011). } \\
\text { 5- (Fritsch y Graf, 2011). } \\
\text { 6- (Maggioni y otros, 2011). } \\
\text { 7- (Yun y Lee, 2013). } \\
\text { 8- (von Proff y Brenner, 2014). } \\
\text { 9- (J. Guan y otros, 2015) }\end{array}$ & $\begin{array}{l}\text { Sistemas } \\
\text { nacionales, } \\
\text { regionales o } \\
\text { sectoriales. }\end{array}$ & $\begin{array}{l}\text { Evidencias de } \\
\text { la influencia de } \\
\text { la proximidad } \\
\text { en el sistema de } \\
\text { innovación. } \\
\text { Evidencias } \\
\text { de fortalezas } \\
\text { según sectores } \\
\text { tecnológicos. }\end{array}$ \\
\hline $\begin{array}{l}\text { Redes de } \\
\text { coautoría } \\
\text { de artículos } \\
\text { científicos }\end{array}$ & $\begin{array}{l}\text { Se revisa la } \\
\text { coautoría } \\
\text { de registros } \\
\text { publicados } \\
\text { en revistas } \\
\text { (Journal) } \\
\text { indexadas en } \\
\text { bases de datos } \\
\text { de corriente } \\
\text { principal. }\end{array}$ & $\begin{array}{l}\text { 10- (Shapiro y otros, 2010). } \\
\text { 11- (Quintella y otros, 2012). } \\
\text { 12- (Binz y otros, 2014). } \\
\text { 13- (J. C. Guan y otros, 2016) }\end{array}$ & $\begin{array}{l}\text { Sistemas } \\
\text { nacionales y de } \\
\text { colaboración } \\
\text { internacional. }\end{array}$ & $\begin{array}{l}\text { Evidencias de } \\
\text { la influencia de } \\
\text { la proximidad } \\
\text { en el sistema de } \\
\text { innovación. }\end{array}$ \\
\hline $\begin{array}{l}\text { Redes de } \\
\text { actores en } \\
\text { proyectos } \\
\text { conjuntos }\end{array}$ & $\begin{array}{l}\text { Se analizan las } \\
\text { redes según } \\
\text { la información } \\
\text { asociada a } \\
\text { los proyectos } \\
\text { conjuntos } \\
\text { desarrollados } \\
\text { entre diferentes } \\
\text { entidades y } \\
\text { agencias de } \\
\text { financiación. }\end{array}$ & $\begin{array}{l}\text { 14- (Boschma y ter Wal, 2007). } \\
\text { 15- (Prats y otros, 2008). } \\
\text { 16- (Steiner y otros, 2010). } \\
\text { 17- (Yan, 2011). } \\
\text { 18- (Kustepeli y otros, 2012). } \\
\text { 19- (Martin y Moodysson, 2013). } \\
\text { 20- (Tsai y otros, 2015). }\end{array}$ & $\begin{array}{l}\text { Muestras de } \\
\text { organizaciones } \\
\text { de sectores } \\
\text { económicos. }\end{array}$ & $\begin{array}{l}\text { Reconocimiento } \\
\text { de flujos } \\
\text { efectivos de } \\
\text { conocimiento } \\
\text { entre actores. }\end{array}$ \\
\hline $\begin{array}{l}\text { Redes } \\
\text { de datos } \\
\text { primarios } \\
\text { obtenidos } \\
\text { mediante } \\
\text { cuestionarios }\end{array}$ & $\begin{array}{l}\text { Se recurre a } \\
\text { los datos de } \\
\text { cuestionarios } \\
\text { para obtener } \\
\text { la información } \\
\text { a partir de las } \\
\text { respuestas de } \\
\text { los diferentes } \\
\text { actores } \\
\text { involucrados } \\
\text { en los sistemas } \\
\text { de innovación }\end{array}$ & $\begin{array}{l}\text { 21- (Sohn y otros, 2009). } \\
\text { 22- (Yokura y otros, 2013). } \\
\text { 23- (Broekel y Brachert, 2015). } \\
\text { 24- (Marek y Blažek, 2016). }\end{array}$ & $\begin{array}{l}\text { Sistemas } \\
\text { nacionales de } \\
\text { innovación. }\end{array}$ & $\begin{array}{l}\text { Reconocimiento } \\
\text { de concentración } \\
\text { de actividades } \\
\text { de innovación } \\
\text { al interior de un } \\
\text { territorio estatal. }\end{array}$ \\
\hline
\end{tabular}




\begin{tabular}{|c|c|c|c|c|}
\hline Categoría & Contenido & Registros (Referencias) & Alcance & Resultados \\
\hline $\begin{array}{l}\text { Redes } \\
\text { analizadas } \\
\text { mediante } \\
\text { datos } \\
\text { combinados }\end{array}$ & $\begin{array}{l}\text { Se usa la } \\
\text { combinación } \\
\text { de } \\
\text { instrumentos, } \\
\text { como son } \\
\text { entrevistas e } \\
\text { información } \\
\text { documental } \\
\text { para analizar } \\
\text { las redes. }\end{array}$ & $\begin{array}{l}\text { 25- (Russo y Rossi, 2009). } \\
\text { 26- (Salavisa y otros, 2012). } \\
\text { 27- (Carson y otros, 2014). } \\
\text { 28- (Niu, 2014). }\end{array}$ & $\begin{array}{l}\text { Sistemas } \\
\text { nacionales o } \\
\text { muy localizados } \\
\text { (regiones, } \\
\text { sectores, } \\
\text { ciudades). }\end{array}$ & $\begin{array}{l}\text { Caracterización } \\
\text { de la red } \\
\text { mediante los } \\
\text { indicadores } \\
\text { asociados } \\
\text { (cohesión, } \\
\text { centralidad). }\end{array}$ \\
\hline $\begin{array}{l}\text { Redes } \\
\text { de datos } \\
\text { mediante } \\
\text { información } \\
\text { en línea } \\
\text { (webometrics) }\end{array}$ & $\begin{array}{l}\text { Se usan } \\
\text { hipervínculos a } \\
\text { los sitios web } \\
\text { de los actores } \\
\text { para analizar } \\
\text { las redes. }\end{array}$ & 29-(Minguillo y Thelwall, 2012). & $\begin{array}{l}\text { Información } \\
\text { en línea de } \\
\text { Parques } \\
\text { Tecnológicos. }\end{array}$ & $\begin{array}{l}\text { Exploración de } \\
\text { la utilidad del } \\
\text { método según } \\
\text { una fuente nueva } \\
\text { de información. }\end{array}$ \\
\hline
\end{tabular}

Fuente: Elaboración propia.

\section{Redes de pateamiento}

Respecto a los procesos asociados a las patentes, se observa la importancia que existe en determinadas tecnologías asociadas a los sistemas de innovación. Es así, que Cantner y otros (2010) resaltan que la información de titulares e inventores funciona como indicadores de transferencia de conocimiento entre actores de los sistemas de información, esto asociado a procesos de colaboración y movilidad laboral. Ahora bien, las patentes no son la única estrategia de innovación, de forma que actividades menos intensivas en protección de invenciones también se encuentran asociadas a la metodología de análisis de redes sociales (Dolfsma y Leydesdorff, 2011).

Estudios que analizan las redes establecidas para la solicitud de patentes en diferentes regiones en Europa (Alemania y Francia principalmente) son presentados en (Cantner y Graf, 2006; Cantner y otros, 2010). Además, se han usado como unidades de estudio los actores del modelo de Triple Hélice (Universidades-Empresa- 
Estado), por ejemplo en la planeación y desarrollo de parques científicos Yun y Lee (2013), el análisis de actividades de co-patentamiento discutido por Breschi y Lissoni (2004) como la solicitud de protección de una invención por medio de patentes por parte de más de una organización o individuo. Por otra parte, se ha usado la información de titulares e inventores para caracterizar redes de co-patentamiento entre regiones al interior de los sistemas de innovación por países (Maggioni y otros, 2011; von Proff y Brenner, 2014). Estas últimas investigaciones difieren en que en la primera se estudian las redes de co-patentamiento de titulares e inventores para establecer la territorialidad de ese aspecto del proceso de innovación al interior de Italia. En el caso del segundo estudio, se usa la información acerca de la ubicación de los inventores para investigar acerca de la territorialidad del proceso de innovación en Alemania.

Guan y otros (2015) utilizan la información de ciudades y países de inventores en el corpus de información de patentes asociadas a energías alternativas, solicitadas en USA (1976-2012), con el fin de lograr comparar y establecer relaciones entre las redes de copatentamiento. De forma similar, se encuentra un análisis de redes que se establecen ente los titulares de patentes, según la información de los documentos de solicitud; asociados a diferentes regiones de Alemania (Fritsch y Graf, 2011)

De otro lado, cabe destacar los resultados del estudio de Dolfsma y Leydesdorff (2011), quienes aplican los métodos asociados al Análisis de Redes Sociales-ARS para identificar la red de tecnologías expresadas en las clasificaciones primarias y secundarias de las patentes según el sistema de la Organización Mundial de la Propiedad Intelectual-OMPI en los Sistemas Nacionales de Innovación de Holanda e India. Para desarrollar su propuesta, estos investigadores analizaron la coocurrencia de las clasificaciones en una muestra de 3287 y 936 patentes de titulares holandeses e indios respectivamente, con el fin de evidenciar las fortalezas nacionales en diversos campos tecnológicos. Mediante la información disponible en patentes, los investigadores citados calculan indicadores de centralidad y de densidad. 


\section{Redes de coautoría de artículos científicos}

En la selección de trabajos de investigación, asociados a las redes de coautoría se evidencia el sesgo hacia una fuente de información específica (Colección Principal de Web of Science-WoS). En este sentido, Quintella y otros (2012) evalúan la coautoría de los investigadores en el área de Química de las universidades públicas del estado de Bahía (Brasil). Por su parte, Binz y otros (2014) analizan el sistema de innovación tecnológico de las instituciones que han publicado artículos asociados a biorreactores de membrana. Guan y otros (2016) discuten y presentan las redes de coautoría desde el nivel de los países para determinar la relación de la intensidad de la colaboración internacional con la eficiencia de las actividades de investigación y desarrollo, en ese mismo nivel de análisis. Por su parte, Shapiro y otros (2010) analizan la ubicación geográfica de los autores afiliados a instituciones de la República de Corea para analizar la colaboración entre provincias y determinar los indicadores de centralidad, densidad y fragmentación de dichas redes.

\section{Redes de datos primarios obtenidos mediante cuestionarios}

Boschma y ter Wal (2007), suministraron a 33 empresas del sector calzado del distrito de Barletta, Italia, entrevistas estructuradas. A los entrevistados se les proporciono un listado de las grandes empresas del sector calzado, para que indicaran de cuál de ellas tomaron conocimientos técnicos o en relación con el mercado, ya sean de clientes, competidores o proveedores. En concordancia con los resultados, se evidencio problemas de desempeño en aquellas empresas que estaban aisladas de las redes de colaboración fundamentadas en conocimiento (Boschma y ter Wal, 2007). Mientras tanto, Kustepeli y otros (2012) realizaron un proceso semejante, al aplicar entrevistas a los gerentes de 37 empresas en Turquía, 20 empresas de la región de Adiyaman y 17 de Denizli, con el objetivo de determinar las relaciones de cooperación en términos de conocimientos técnicos o el mercado en el sector textil. 
Por su parte, Prats y otros (2008) por medio de entrevistas a actores destacados del sector turístico de la Costa Brava, España, estudian el sistema local de innovación; estos actores fueron identificados a través del análisis de sitios web locales y otras fuentes. Entretanto, Steiner y otros (2010), para recopilar información sobre los proveedores y socios de actividades de Investigación y Desarrollo $(I+D)$, se entrevistaron con empresas de las siguientes regiones europeas: 1) Estiria, 2) Gales, 3) Hamburgo; y 4) Madrid). De forma similar, Yan (2011) mediante entrevistas a miembros de su unidad de estudio, ya sean líderes u otros, analiza la red de asesoría de dos grupos de investigación en China.

Por otra parte, Martin y Moodysson (2013) también realizaron entrevistas como metodología para determinar los flujos de conocimiento de las empresas con otros actores, en total, entrevistaron a informantes de 95 empresas del sur de Suecia. Por medio de las entrevistas, los autores buscaron analizar las redes regionales de cooperación de empresas de los siguientes tres sectores económicos: 1) Ciencias de la vida, 2) Alimentos de comunicación; y 3) Medios de comunicación; en concordancia con una categorización de las bases de conocimientos relacionadas a cada sector económico, siendo estas tres: 1) Base analítica, 2) Base sintética; y 3) Base simbólica. Cabe mencionar, que esta metodología es similar a la de Marek y Blažek (2016). Aún más, Tsai y otros (2015) utilizaron una muestra de 12 empresas en Taiwan, para realizar un estudio de las redes internacionales en biotecnología relacionadas con los bancos de sangre del cordón umbilical. Para este estudio, fue importante calcular la centralidad eigenvector y de los hoyos estructurales, los investigadores lo hallaron efectivamente relacionado con el establecimiento de alianzas internacionales de cooperación (Tsai y otros, 2015).

\section{Redes de actores en proyectos conjuntos}

Según Broekel y Brachert (2015), usar esta fuente de datos admite envolver diversos sectores además de aquellos en donde se protege la propiedad intelectual con gran magnitud mediante patentes. 
Autores como Yokura y otros (2013), para examinar la cooperación de los actores del Sistema Nacional de Japón de acuerdo con el esquema de la Triple Helice y las divisiones regionales administrativas, usan los datos de proyectos entregados al Ministerio de Economía, Comercio e Industria (METI) de dicho país. Por su parte, Sohn y otros (2009) para determinar la injerencia de universidades en sus lazos con el sector productivo, realizaron un análisis de las respuestas, obtenidas por el Instituto de Política Científica y Tecnológica, a la encuesta de Innovación de la República de Corea, encuesta que fue aplicada a empresas de los clústeres de innovación de Gumi y Ulsan.

Por otra parte, Marek y Blažek (2016) se encargaron de analizar las interacciones de cooperación entre dos grandes actores de la Tripe Helice, en este caso, empresas y universidades, coincidiendo con la información de los proyectos de Investigación y Desarrollo I+D los cuales son financiados por el Estado checo. Estos autores concluyen, desde un punto de vista geográfico, que existe una concentración en la capital de este país relacionada con la presentación de estos proyectos conjuntos. Cabe destacar, que estos autores como se mencionó en la metodología de Martin y Moodysson (2013), aplicaron una categorización de los proyectos asociado al tipo de base de conocimiento: 1) Base analítica, 2) Base sintética; y 3) Base simbólica, con el fin de representar la red de cooperación con los datos disponibles.

Aún más, Broekel y Brachert (2015) a través de los datos de los proyectos de cooperación subsidiados por el gobierno alemán, realizan un análisis acerca de la complementariedad entre sectores económicos. Pese a los sesgos existentes en la recopilación de datos, los actores concluyen que la información oficial tiene una cobertura que les permite comprender sectores menos habituales en el proceso de petición de patentes (Broekel y Brachert, 2015).

\section{Redes analizadas mediante datos combinados}

En Italia, exactamente en la región de Toscana, Russo y Rossi (2009) usaron datos del 2001 a 2004 del programa "Innovazione Tecno- 
logica in Toscana" para su investigación. Esta iniciativa tenía como objetivo estimular a la innovación a través de la transferencia de desarrollos tecnológicos y tecnología, todo esto orientado al desarrollo económico de dicha región mediante la labor de cooperación entre empresas y entidades de investigación. Además, los autores realizaron entrevistas a los agentes de las empresas y universidad que participaron en el programa, para complementar la información administrativa del mismo.

Por su parte, Salavisa y otros (2012) realizaron un estudio de redes a una muestra de 52 empresas portuguesas de biotecnología y software, a través de la obtención de datos documentales y datos de las entrevistas semiestructuradas aplicadas a los informantes de cada una de las empresas de la muestra.

Carson y otros (2014) con el objetivo de examinar las redes de cooperación en Clare Valley Australia, destino turístico, se encargaron de combinar fuentes documentales en línea, 30 entrevistas con los representantes de los actores de su unidad de estudio, planes estratégicos de las empresas de la muestra, noticias en periódicos y observaciones de campo logradas en las reuniones de los actores del sector en estudio.

Aún más, Niu (2014) realizó dos fases metodológicas, para analizar la red de cooperación científica entre investigadores de los Sistemas Nacionales de Innovación de Australia y la República Popular de china. La primera fase, se basó en el análisis bibliométrico de la coautoría de los artículos indexados en el Science Citation Index-Expanded y Scopus publicados entre 1981 y 2010; la segunda, se basó en la realización de entrevistas a 79 investigadores, identificados a través del análisis bibliométrico, de los países estudiados.

\section{Redes de datos mediante información en línea (webometrics)}

Minguillo y Thelwall (2012) utilizaron el software SocSciBot para estudiar los hipervínculos desde y hacia los sitios web de los siguientes tres parques tecnológicos en el Reino Unido: 1) Advanced 
Manufacturing Park, 2) Leeds Innovation Centre; y 3) York Science Park. Además, mediante este método, realizaron un estudio de cinco medidas relacionadas con las redes: 1) a saber, 2) inclusividad, 3) conectividad, 4) densidad; y 5) reciprocidad.

\section{Conclusiones}

En el presente estudio se pretendió responder a la pregunta acerca del cómo el Análisis de Redes Sociales ha sido aplicado en los estudios sobre la innovación a Sistemas Nacionales, Regionales y Locales. En tal orden de ideas, se prestó especial atención a los aspectos metodológicos de los estudios incluidos en la revisión con el fin de determinar los alcances y los resultados de los métodos identificados en dichas publicaciones.

El análisis de redes sociales se ha aplicado a los sistemas de innovación con el propósito de entender su eficiencia por medio de la evaluación de la intensidad de las relaciones de sus actores y la influencia de variables como la proximidad geográfica, evidenciadas de forma preferencial a partir de fuentes de información secundaria, como patentes y artículos científicos.

Mediante la metodología de consulta y análisis de la información en las bases de datos de corriente principal, se consolidó un corpus de artículos científicos con los cuales es posible profundizar en la comprensión de las aplicaciones del Análisis de Redes Sociales a Sistemas de Innovación. De acuerdo con el análisis bibliométrico, se evidenciaron fortalezas de los investigadores en países con economías fuertemente basadas en el conocimiento, tales como Estados Unidos, Holanda, Reino Unido, Italia, Suecia, entre otros, tanto desde el punto de vista de la cantidad de publicaciones como desde el punto de vista del impacto reflejado en la cantidad de citas recibidas.

Por otra parte, la exploración de las palabras clave incluidas en cada registro permitió detectar temáticas de investigación con ma- 
yor cercanía según la frecuencia de la coocurrencia. La representación de tal red de conceptos permitió evidenciar la relevancia de temas de investigación agrupados en categorías temáticas más generales. La revisión sistemática de los 29 referentes incluidos permitió inferir que cada fuente aplicada para el análisis de redes sociales en sistemas de innovación (patentes, artículos científicos, información primaria, información de proyectos, datos combinados, o información webométrica) tiene ventajas y limitaciones en cuanto a la cobertura de la información que ofrece, en términos de cantidad de datos, sectores o actividades económicas incluidas, la posibilidad de generalizar a partir de las conclusiones, entre otras.

Finalmente, hay que reconocer como limitaciones del presente estudio, entre otros aspectos, los alcances de las fuentes elegidas para la obtención de la información, debido a que es probable que haya fuentes pertinentes por fuera de las bases de datos de corriente principal que hubieran enriquecido el cumplimiento de los objetivos de la revisión.

\section{Agradecimientos}

Los autores quieren agradecen el aporte al equipo del Doctorado en educación de la Universidad Santo Tomás-Colombia, de la Maestría en gerencia de la innovación y el conocimiento de la Universidad Industrial de Santander-Colombia y del Centro de Recursos para el Aprendizaje y la Investigación-CRAI de la Universidad Santo Tomás-Colombia.

\section{Referencias bibliográficas}

Acevedo-Argüello, C., Zabala-Vargas, S., \& Guayán-Perdómo, O. (2018). El análisis de redes sociales como herramienta para estudiar los sistemas de innovación: Una revisión sistemática de la literatura. Universidad Industrial de Santander. 
Balconi, M., Breschi, S., \& Lissoni, F. (2004). Networks of inventors and the role of academia: an exploration of Italian patent data. Research Policy, 33, 127-145. https://doi.org/10.1016/S0048-7333(03)00108-2

Benito-Crosetti, B., Ardila-Segovia, D., Zabala-Vargas, S., \& García-Mora, L. (2019). Aprendizaje Basado en Juegos (GBL) aplicado a la enseñanza de la matemática en educación superior: Una revisión sistemática de literatura. Formación Universitaria, 12(4).

Binz, C., Truffer, B., \& Coenen, L. (2014). Why space matters in technological innovation systems - Mapping global knowledge dynamics of membrane bioreactor technology. Research Policy, 43(1), 138-155. https://doi.org/10.1016/j.respol.2013.07.002

Borgatti, S. P., Everett, M. G., \& Johnson, J. C. (2013). Analyzing Social Networks. SAGE Publications. Retrieved from https://books.google.com. co/books?id=VjS6NAEACAAJ

Boschma, R. A., \& ter Wal, A. L. J. (2007). Knowledge Networks and Innovative Performance in an Industrial District: The Case of a Footwear District in the South of Italy. Industry \& Innovation, 14(2), 177-199. https://doi.org/10.1080/13662710701253441

Breschi, S., \& Lissoni, F. (2004). Knowledge networks from patent data. In H. F. Moed, W. Glänzel, \& U. Schmoch (Eds.), Handbook of Quantitative Science and Technology Research (pp. 613-643). Dordrecht: Springer. https://doi.org/10.1007/1-4020-2755-9_29

Broekel, T., \& Brachert, M. (2015). The Structure and Evolution of Intersectoral Technological Complementarity in R\&D in Germany from 1990 to 2011. Journal of Evolutionary Economics, 25, 755-785. https:// doi.org/10.1007/s00191-015-0415-7

Cantner, U., \& Graf, H. (2006). The network of innovators in Jena: An application of social network analysis. Research Policy, 35(4), 463-480. https://doi.org/10.1016/J.RESPOL.2006.01.002 
Cantner, U., Meder, A., \& ter Wal, A. L. J. (2010). Innovator networks and regional knowledge base. Technovation, 30(9-10), 496-507. https://doi. org/10.1016/J.TECHNOVATION.2010.04.002

Carson, D. A., Carson, D. B., \& Hodge, H. (2014). Understanding local innovation systems in peripheral tourism destinations. Tourism Geographies, 16(3), 457-473. https://doi.org/10.1080/14616688.2013 .868030

Clarivate Analytics. (2018). Web of Science. Retrieved June 5, 2018, from https://clarivate.com/products/web-of-science/

Davyt, A., \& Mujica, A. (2011). La promoción de redes de innovación como componente central de una estrategia política de vinculación del conocimiento y el desarrollo. Hallazgos, 8(16), 19-28.

de Souza, L. G. A., de Moraes, M. A. F. D., Dal Poz, M. E. S., \& da Silveira, J. M. F. J. (2015). Collaborative Networks as a measure of the Innovation Systems in second-generation ethanol. Scientometrics, 103(2), 355-372. https://doi.org/10.1007/s11192-015-1553-2

Dolfsma, W., \& Leydesdorff, L. (2011). Innovation systems as patent networks: The Netherlands, India and Nanotech. Innovation: Management, Policy and Practice, 13(3). https://doi.org/10.5172/ impp.2011.13.3.311

Doloreux, D., \& Porto Gomez, I. (2017). A review of (almost) 20 years of regional innovation systems research. European Planning Studies, 25(3), 371-387. https://doi.org/10.1080/09654313.2016.1244516

Edquist, C. (2006). Systems of Innovation: Perspectives and Challenges. In J. Fagerberg, D. C. Mowery, \& R. R. Nelson (Eds.), The Oxford Handbook of Innovation (pp. 181-208). Nueva York: Oxford University Press. https://doi.org/10.1093/oxfordhb/9780199286805.003.0007

Elsevier. (2018). Scopus. Retrieved June 5, 2018, from https://www. scopus.com/home.uri 
Fernández Chaves, F. (2002). El análisis de contenido como ayuda metodológica para la investigación. Revista de Ciencias Sociales (Cr), II(96). Retrieved from https://www.redalyc.org/articulo.oa?id=15309604

Fritsch, M., \& Graf, H. (2011). How sub-national conditions affect regional innovation systems: The case of the two Germanys. Papers in Regional Science, 90(2), 331-353. https://doi.org/10.1111/j.14355957.2011.00364.x

Galeano, C., Bellón, D., Zabala, S., Romero, E., \& Duro, V. (2017). Identificación de los pilares que direccionan a una institución universitaria hacia un Smart-campus. In CITED 2017.

Gast, I., Schildkamp, K., \& van der Veen, J. T. (2017). Team-Based Professional Development Interventions in Higher Education: A Systematic Review. Review of Educational Research, 87(4), 736-767. https://doi.org/10.3102/0034654317704306

Guan, J. C., Zuo, K. R., Chen, K. H., \& Yam, R. C. M. (2016). Does country-level R\&D efficiency benefit from the collaboration network structure? Research Policy, 45(4), 770-784. https://doi.org/10.1016/J. RESPOL.2016.01.003

Guan, J., Zhang, J., \& Yan, Y. (2015). The impact of multilevel networks on innovation. Research Policy, 44(3), 545-559. https://doi.org/10.1016/j. respol.2014.12.007

Kustepeli, Y., Gulcan, Y., \& Akgungor, S. (2012). The innovativeness of the Turkish textile industry within similar knowledge bases across different regional innovation systems. European Urban and Regional Studies, 20(2), 227-242. https://doi.org/10.1177/0969776412448088

Lundvall, B. (2007). National Innovation Systems-Analytical Concept and Development Tool. Industry \& Innovation, 14(1), 95-119. https:// doi.org/10.1080/13662710601130863

Maggioni, M. A., Uberti, T. E., \& Usai, S. (2011). Treating patents as relational data: Knowledge transfers and spillovers across Italian 
provinces. Industry and Innovation, 18(1), 39-67. https://doi.org/10.10 $80 / 13662716.2010 .528928$

Marek, D., \& Blažek, J. (2016). The challenge of breaking the academiabusiness firewall in Czechia: comparing the role of differentiated knowledge bases in collaborative R\&D projects. European Planning Studies, 24(4), 809-831. https://doi.org/10.1080/09654313.2015.1121 204

Martin, R., \& Moodysson, J. (2013). Comparing knowledge bases: on the geography and organization of knowledge sourcing in the regional innovation system of Scania, Sweden. European Urban and Regional Studies, 20(2), 170-187. https://doi.org/10.1177/0969776411427326

Minguillo, D., \& Thelwall, M. (2012). Mapping the network structure of science parks. Aslib Proceedings, 64(4), 332-357. https://doi. org/10.1108/00012531211244716

Niu, X. S. (2014). International scientific collaboration between Australia and China: A mixed-methodology for investigating the social processes and its implications for national innovation systems. Technological Forecasting and Social Change, 85, 58-68. https://doi.org/10.1016/j. techfore.2013.10.014

OECD/Eurostat. (2005). Oslo Manual: Guidelines for Collecting and Interpreting Innovation Data, 3rd Edition, The Measurement of Scientific and Technological Activities. Paris. https://doi. org/10.1787/9789264013100-en.

OECD. (2014). The Innovation Imperative Contributing to Productivity, Growth and Well-Being. Paris. Retrieved from https:// www.oecd-ilibrary.org/science-and-technology/the-innovationimperative_9789264239814-en/citation

Petticrew, M., \& Roberts, H. (2006). Systematic Reviews in the Social Sciences: A Practical Guide. (Oxford, Ed.). Wiley-Blackwell.

Pinheiro, M. L., Lucas, C., \& Pinho, J. C. (2015). Social Network Analysis as a New Methodological Tool to Understand University - Industry 
Cooperation. International Journal of Innovation Management, 19(01), 1550013. https://doi.org/10.1142/S1363919615500139

Prats, L., Guia, J., \& Molina, F. X. (2008). How tourism destinations evolve: The Notion of Tourism Local Innovation System. Tourism and Hospitality Research, 8(3), 178-191. https://doi.org/10.1057/thr.2008.24

Quintella, R. H., da Silva Monteiro de Freitas, E. J., Ventura, A. C., Santos, M. A., de Melo, D. R. A., da Silva Motta, G., \& Jucá, S. (2012). Scientific knowledge networks in peripheral regions and local innovation systems: The case of chemistry in the state of Bahia. Journal of Technology Management and Innovation, 7(1), 85-103.

Russo, M., \& Rossi, F. (2009). Cooperation Networks and Innovation. Evaluation, 15(1), 75-99. https://doi.org/10.1177/1356389008097872

Salavisa, I., Sousa, C., \& Fontes, M. (2012). Topologies of innovation networks in knowledge-intensive sectors: Sectoral differences in the access to knowledge and complementary assets through formal and informal ties. Technovation, 32(6), 380-399. https://doi.org/10.1016/j. technovation.2012.02.003

Schiller, M. C. O. S. (2015). A survey of the national innovation system. Global Business and Economics Review, 17(3), 310. https://doi. org/10.1504/GBER.2015.070308

Shapiro, M. A., So, M., \& Park, H. W. (2010). Quantifying the national innovation system: Inter-regional collaboration networks in South Korea. Technology Analysis and Strategic Management, 22(7), 845-857. https://doi.org/10.1080/09537325.2010.511158

Smith, H. L., \& Leydesdorff, L. (2014). The Triple Helix in the context of global change: dynamics and challenges. Prometheus, 32(4), 321-336. https://doi.org/10.1080/08109028.2014.972135

Sohn, D. W., Kim, H., \& Lee, J. H. (2009). Policy-driven university - Industry linkages and regional innovation networks in Korea. Environment 
and Planning C: Government and Policy, 27(4), 647-664. https://doi. org/10.1068/c0890b

Steiner, M., Gil, J. A., Ehret, O., Ploder, M., \& Wink, R. (2010). European medium-technology innovation networks: a multi-methodological multi-regional approach. Int. J. Technology Management, 50(3/4), 229262.

Stuck, J., Broekel, T., \& Revilla Diez, J. (2016). Network Structures in Regional Innovation Systems. European Planning Studies, 24(3), 423442. https://doi.org/10.1080/09654313.2015.1074984

Swar, B., \& Khan, G. F. (2013). An analysis of the information technology outsourcing domain: A social network and Triple helix approach. Journal of the American Society for Information Science and Technology, 64(11), 2366-2378. https://doi.org/10.1002/asi.22918

Tsai, H.-T. T., Huang, S.-Z. Z., \& Wang, C.-H. H. (2015). Cross-border R\&D alliance networks: An empirical study of the umbilical cord blood banking industry in emerging markets. Asian Journal of Technology Innovation, 23(3), 383-406. https://doi.org/10.1080/19761597.2015.1120502

Van Der Valk, T., \& Gijsbers, G. (2010). The use of social network analysis in innovation studies: Mapping actors and technologies. Innovation, 12(1), 5-17. https://doi.org/10.5172/impp.12.1.5

von Proff, S., \& Brenner, T. (2014). The dynamics of inter-regional collaboration: An analysis of co-patenting. Annals of Regional Science, 52(1), 41-64. https://doi.org/10.1007/s00168-013-0573-1

Waltman, L., \& Noyons, E. (2018). Bibliometrics for Research Management and Research Evaluation. Leiden, The Netherlands: CWTS, B.V. Retrieved from http://www.cwts.nl/pdf/CWTS_bibliometrics.pdf

Yan, J. (2011). A study on consulting network of university research team. In 2011 2nd International Conference on Artificial Intelligence, Management Science and Electronic Commerce (AIMSEC) (pp. 18711874). IEEE. 
Yokura, Y., Matsubara, H., \& Sternberg, R. (2013). R\&D networks and regional innovation: A social network analysis of joint research projects in Japan. Area, 45(4), 493-503. https://doi.org/10.1111/area.12055

Yun, S., \& Lee, J. (2013). An innovation network analysis of science clusters in South Korea and Taiwan. Asian Journal of Technology Innovation, 21(2), 277-289. https://doi.org/10.1080/19761597.2013.8 66310

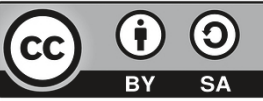

